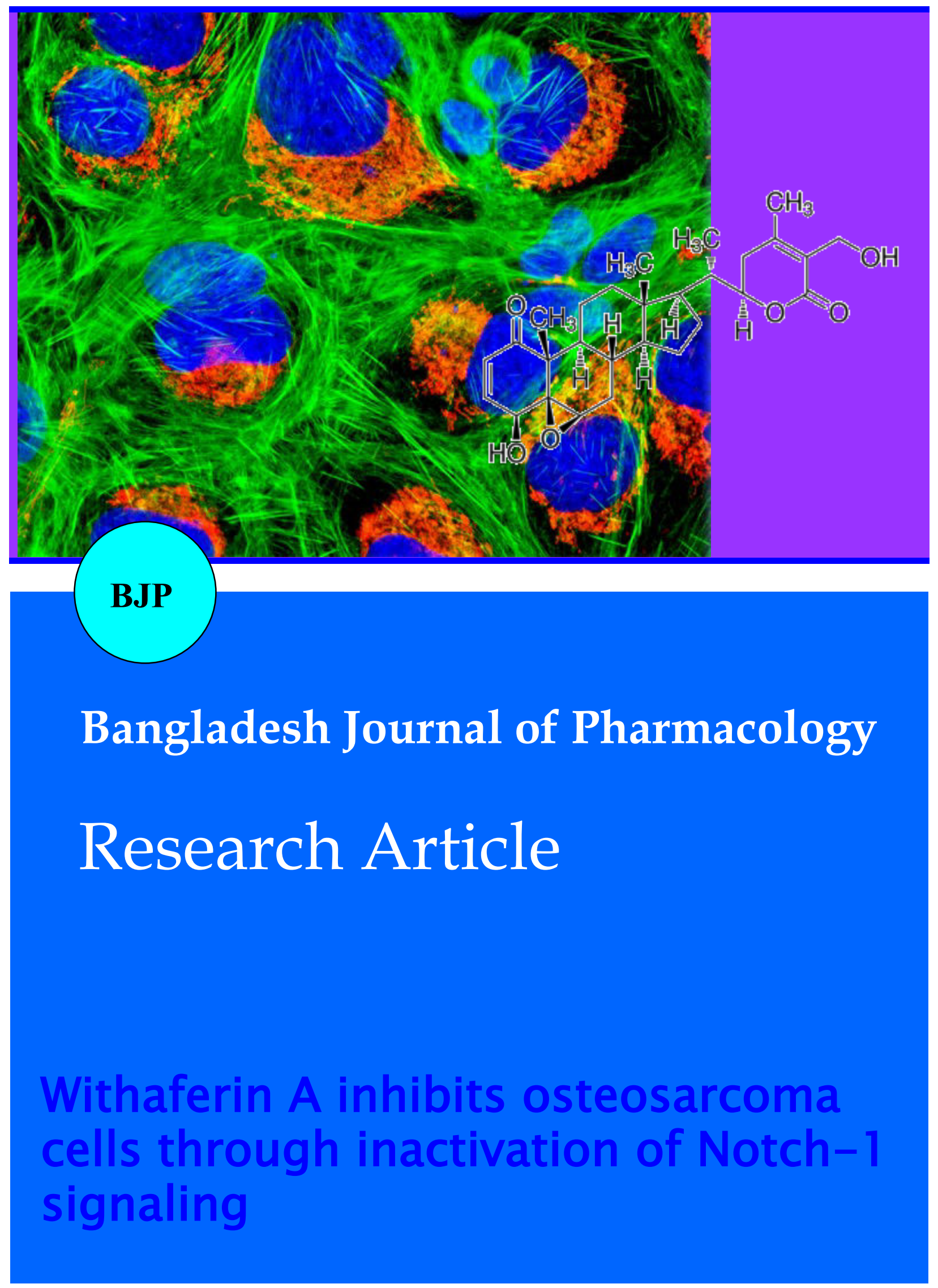




\title{
Withaferin A inhibits osteosarcoma cells through inactivation of Notch-1 signaling
}

\author{
Yang Chen, Xiang Zhen Han, Wei Wang, Ren Tao Zhao and Xiao Li \\ Department of Bone Tumor, Linyi People's Hospital, Shandong 276003 , China.
}

\begin{tabular}{|c|c|}
\hline Article Info & \\
\hline Received: & 18 July 2014 \\
\hline Accepted: & 27 July 2014 \\
\hline Available Online: & 19 August 2014 \\
\hline DOI: $10.3329 /$ bjp. & .19568 \\
\hline Cite this article: & \\
\hline Chen $Y$, Han XZ, & ing W, Zhao R] \\
\hline $\begin{array}{l}\text { Li X. Withaferin } \\
\text { coma cells throt }\end{array}$ & $\begin{array}{c}\text { nhibits osteosar } \\
\text { inactivation }\end{array}$ \\
\hline $\begin{array}{l}\text { Notch-1 signaling } \\
\text { macol. } 2014 ; 9: 36\end{array}$ & ngladesh J Pha \\
\hline
\end{tabular}

\section{Article Info}

Received:

\begin{abstract}
Human osteosarcoma cells were treated with withaferin A and the antiproliferative activity was evaluated by the 3-(4, 5 dimethyl-thiazol-2-yl)-2, 5diphenyltetrazolium bromide (MTT) assays. Real-time RT-PCR and western blot were used to investigate whether the down-regulation of Notch-1 contributes to withaferin A-induced inhibition of proliferation in osteosarcoma cells. The results showed that withaferin A caused marked inhibition of osteosarcoma cell growth and G2M phase cell cycle arrest. This was associated with concomitant attenuation of Notch-1 and down-regulation of its downstream genes, such as matrix metalloproteinases. These results suggest that antitumor activity of withaferin A is mediated through a novel mechanism involving inactivation of the Notch-1 signaling pathway. Our data provide the first evidence that the down regulation of Notch-1 by withaferin A may be an effective approach for the treatment of osteosarcoma.
\end{abstract}

\section{Introduction}

Osteosarcoma, a primary malignancy which predominantly affects rapidly growing bones in adolescents and children, is characterized by locally aggressive growth and early metastatic potential (Arndt and Crist, 1999). Despite significant clinical improvements through the use of combination intensive chemotherapy and surgical resection, respiratory failures due to lung metastases are common events and the major cause of death in these patients (Petrilli et al., 2006; Bielack et al., 2002). Moreover, effective therapeutic options available for patients who either relapse following administration of currently approved chemotherapeutic agents or suffer intolerable acute and long term toxicities from chemotherapy treatments are seriously lacking. Therefore, more effective treatment strategies are urgently needed to prevent osteosarcoma disease progression and improve patient survival rates.

It is well established that various compounds of Withania species, such as withaferin A from the leaves
(Jayaprakasam et al., 2003), and ashwagandhanolides from the roots of $W$. somnifera (Subbaraju et al., 2006) are known to possess anti-cancer properties. The mechanism by which withanolides demonstrate antiproliferative, antimetastatic, antiangiogenic, anti-invasive, and proapoptotic activities has been associated with the suppression of NF-jB and NF-jB-regulated gene products (Ichikawa et al., 2006). It was shown that WA inhibits nuclear factor-kB (NF-kB) activation and induces apoptosis in prostate cancer cells through Par-4 induction (Srinivasan et al. 2007), inhibits IkB kinase activation via a thioalkylation-sensitive redox mechanism (Kaileh et al., 2007).

The Notch signaling pathway plays a pivotal role in fundamental processes of cell-fate determination, including stem cell maintenance, differentiation, proliferation and apoptosis (Li and Harris, 2005), and thereby may contribute to the carcinogenesis of osteosarcoma. Deregulated Notch receptor expression has been reported in a growing number of solid human tumors (Leong and Karsan, 2006), such as pancreas (Wang et al., 2006), 
cervix (Ramdass et al., 2007) and colon (Katoh, 2007), as well as osteosarcoma (Engin et al., 2009). It has also been reported that manipulation of Notch-1 showed a crucial role for Hes-1 in osteosarcoma invasion and metastasis (Zhang et al., 2008). Moreover, inhibition of the Notch pathway using c-secretase inhibitor and CBF1 small-interfering RNA (siRNA) suppressed osteosarcoma growth in vitro and in vivo (Tanaka et al 2009). Therefore, the down-regulation of Notch signaling may be an effective approach for osteosarcoma therapy. In this study, antiproliferative effects of WA have been studied in two osteosarcoma cell lines namely (U2OS and MG-63). The mechanism of action of cell cycle arrest and apoptosis were also investigated.

\section{Materials and Methods}

\section{Reagents}

Bovine serum albumin (BSA), ribonuclease A (RNase $\mathrm{A})$, propidium iodide (PI), trichloroacetic acid (TCA), sulforhodamine B (SRB), anti- $\beta$-actin antibody and withaferin A were purchased from Sigma (St.Louis, MO, USA). fetal bovine serum (FBS), RPMI 1640, trypsin-EDTA and antibiotic-antimycotic solution were purchased from GIBCO-BRL (Grand Island, NY, USA). Primary antibodies for Notch-1 intracellular domain and Hes-1 were purchased from Abcam (Cambridge, UK). Primary antibodies for cyclin D1, MMP-2 and MMP-9 were obtained from Cell Signaling Technology (Beverly, MA, USA).

\section{Cell lines and culture}

Human osteosarcoma cell lines MG-63, U2OS and MG63 were obtained from the China Center for Type Culture Collection (CCTCC). The cells were cultured with high glucose DMEM supplemented with 10\% FBS, $100 \mathrm{U} / \mathrm{mL}$ of penicillin $\mathrm{G}$ and $100 \mathrm{U} / \mathrm{mL}$ of streptomycin. They were cultured at $37^{\circ} \mathrm{C}$ in a humidified incubator with $5 \% \mathrm{CO}_{2}$. All cells used in the studies were in the logarithmic phase.

\section{Cell cytotoxicity assay}

U2OS, SaOS-2 and MG-63 cells $\left(8 \times 10^{3}, 5 \times 10^{3}\right.$ and $6 \times$ $10^{3}$, respectively) were seeded in a 96-well culture plate. After 12 hours, cells were treated with 10, 20, 30 and 40 $\mu \mathrm{M}$ of withaferin A for 24, 48 and 72 hours. Control cells were treated with $0.1 \%$ dimethyl-sulfoxide in culture medium. After treatment, the cells were incubated with MTT solution $(5 \mathrm{mg} / \mathrm{mL}$ in phosphate-buffered saline, $\mathrm{NaCl} / \mathrm{Pi}$ ) at $37^{\circ} \mathrm{C}$ for 4 hours and then with dimethylsulfoxide by shaking at room temperature for $10 \mathrm{~min}$. The spectrophotometric absorbance was measured at $570 \mathrm{~nm}$ on a multifunctional microplate reader (Tecan, USA). Cell viability was expressed as the relative formazan formation in treated samples compared with control cells after correction for background absorbance. The $\mathrm{IC}_{50}$ values, defined as the concentration inducing $50 \%$ loss of cell viability, were estimated from the dose-response curves. Results were plotted as the mean \pm SD of three separate experiments from six determinations per experiment for each experimental condition.

\section{Cell cycle analysis}

U2OS, SaOS-2 and MG-63 cells were plated at density of $1 \times 10^{6}$ cells per $100 \mathrm{~mm}$ culture dish and incubated for 24 hours. We replaced the medium with fresh medium containing various concentrations of WA $(1,10$ and $100 \mu \mathrm{M}$ ) and $0.1 \%$ DMSO alone. Following 24 hours of treatment at $37^{\circ} \mathrm{C}$, all cells were collected, washed with phosphate-buffered saline (PBS), and fixed with $70 \%(\mathrm{v} / \mathrm{v})$ ice-cold ethanol for 1 hour at $4^{\circ} \mathrm{C}$. The fixed cells were treated with $50 \mu \mathrm{g} / \mathrm{mL}$ RNase A and $50 \mu \mathrm{g} /$ $\mathrm{mL}$ PI for $30 \mathrm{~min}$ at room temperature. At least 10,000 cells were analyzed by flow cytometry (FACSCalibur, Becton Dickinson, USA). Data analysis was performed using Cell Quest cell cycle analysis software.

\section{Western blot analysis}

U2OS and MG-63 human osteocarcinoma cells were exposed to various concentrations of WA for 24 hours. After incubation, cells were lysed and protein concentrations were determined by Lowry method. Cell samples were lysed in sample buffer $(150 \mathrm{mM}$ Tris $\mathrm{pH}$ 6.8, $8 \mathrm{M}$ urea, $50 \mathrm{mM}$ DTT, $2 \%$ sodium dodecyl sulfate, $15 \%$ sucrose, 2 mM EDTA, $0.01 \%$ bromophenol blue, and $1 \%$ protease and phosphatase inhibitor cocktails), sonicated and protein concentrations were determined by Lowry method. The samples (40-45 $\mu \mathrm{g}$ ) were boiled, run through an $8-12 \%$ Bis/Tris gel using $5 \times$ MES buffer (Invitrogen), transferred to an Immobilon NC membrane (Millipore) and blocked in 5\% non-fat milk in TBST (150 mM NaCl, $50 \mathrm{mM}$ Tris $\mathrm{pH}$ 7.5 , and $0.1 \%$ Tween-20). The membranes were then incubated with appropriate primary antibodies (Notch-1, Hes-1, cyclin D1,MMP-2 and MMP-9), followed by incubation with horseradish peroxidase conjugated anti-rabbit IgG (1:5000) in blocking buffer at room temperature for $90 \mathrm{~min}$, followed by washing. Immunoreactive bands were detected by enhanced chemiluminescence (GE Healthcare, UK).

\section{Real-time PCR assay}

RT-PCR is carried out to analyse the change at the transcriptional levels. Briefly, MG-63 and U2OS cancer cells are treated with $20 \mu \mathrm{M}$ WA for 12 hours. TRIzol reagents (Invitrogen, Carlsbad, CA) are used to extract total cellular RNAs as described in protocol provided by manufacturer. Superscript III first strand synthesis kit from invitrogen is used to reverse transcribe the cDNA. Then the real-time PCR is carried out in ABI PRISM 7900T real-time PCR system (PerkinElmer, Branchburg, NJ) with SYBR Green PCR Master Mix (Applied Biosystems, USA). The primers 
Table I

Primer sequences used in RT-PCR analysis

\begin{tabular}{lcc} 
Gene & Forward primes $\left(5^{\prime}-3^{\prime}\right)$ & Reverse primes $\left(5^{\prime}-3^{\prime}\right)$ \\
\hline Notch-1: & TCAGCGGGATCCACTGTGAG & ACACAGGCAGGTGAACGAGTTG \\
Hes-1: & GAGCACAGAAAGTCATCAAAGC & ATTTCCAGAATGTCCGCCTTC \\
Hey-1 & CGGAGAGGAATAATTGAGAAGCG & CTTTCCCTCCTGCCGTATGC \\
Hey-2 & TTCAAGGCAGCTCGGTAACT & GGGCATTTTACTTCCCCAAT \\
MMP-2 & CAGGGAATGAATACTGGATCTACT & GCTCCAGTTAAAGGCGGCATCCAC \\
MMP-9 & GCCTGCAACGTGAACATCT & TCAAAGACCGAGTCCAGCTT \\
GAPDH & GCACCGTCAAGGCTGAGAAC & TGGTGAAGACGCCAGTGGA
\end{tabular}

used in RT-PCR are shown in Table I. mRNA levels are calculated as fold change of control. After completion of the RT-PCR, Ct values (cycle numbers in which signal intensity equal to the threshold value) will be obtained from the software. For each samples, $\Delta \mathrm{Ct}$ is calculated as $\Delta \mathrm{Ct}=\mathrm{CtAkt} / \mathrm{Cdk} 4 / \mathrm{GR}-$ Ctactin. Then $\Delta \Delta \mathrm{Ct}$ is calculated as $\Delta \Delta \mathrm{Ct}=\Delta \mathrm{Cttreatment}$ $\Delta$ Ctcontrol. The fold change of the Akt/Cdk4/GR mRNA levels relative to control group are calculated as $2-\Delta \Delta \mathrm{Ct}$.

\section{Statistical analysis}

All experiments were performed in duplicate and repeated at least three times. The data are expressed as the means \pm standard deviation (SD). The treatment groups were compared by one-way variance (ANOVA) with SPSS 12.0 (SPSS Inc., Chicago). Differences were considered statistically significant at $\mathrm{p}<0.05$.

\section{Results}

The effect of a drug can be cytotoxic or cytostatic. In initial experiments, the cytotoxic effect of withaferin A on osteosarcoma cell lines, namely U2OS, SaOS-2 and MG-63, was evaluated using 3-(4,5-dimethylthiazol-2yl)-2,5-diphenyltetrazolium bromide (MTT) assays. Withaferin A reduced the proliferation of these cells in a dose-dependent manner (Figure 1). The half-maximal inhibitory concentration $\left(\mathrm{IC}_{50}\right)$ of withaferin $\mathrm{A}$ at 72 hours was found to be $20.1 \pm 1.2,20.0 \pm 1.1$ and $21.7 \pm$ $1.2 \mu \mathrm{M}$ in U2OS, SaOS-2 and MG-63 cells, respectively. To determine an optimum (effective yet non-toxic) dose range for subsequent studies, time response cytotoxic assays were performed next. Our data showed that treatment of osteosarcoma cells with withaferin A beyond $30 \mu \mathrm{M}$ at 24,48 and 72 hours resulted in statistically significant toxicities and hence the maximum concentration employed in subsequent studies was limited to $20 \mu \mathrm{M}$ (Figure 1).

As discussed above, treatment of U2OS, SaOS-2 and MG-63 cells with increasing concentrations of withaferin $A$ resulted in a dose-dependent decrease in viable

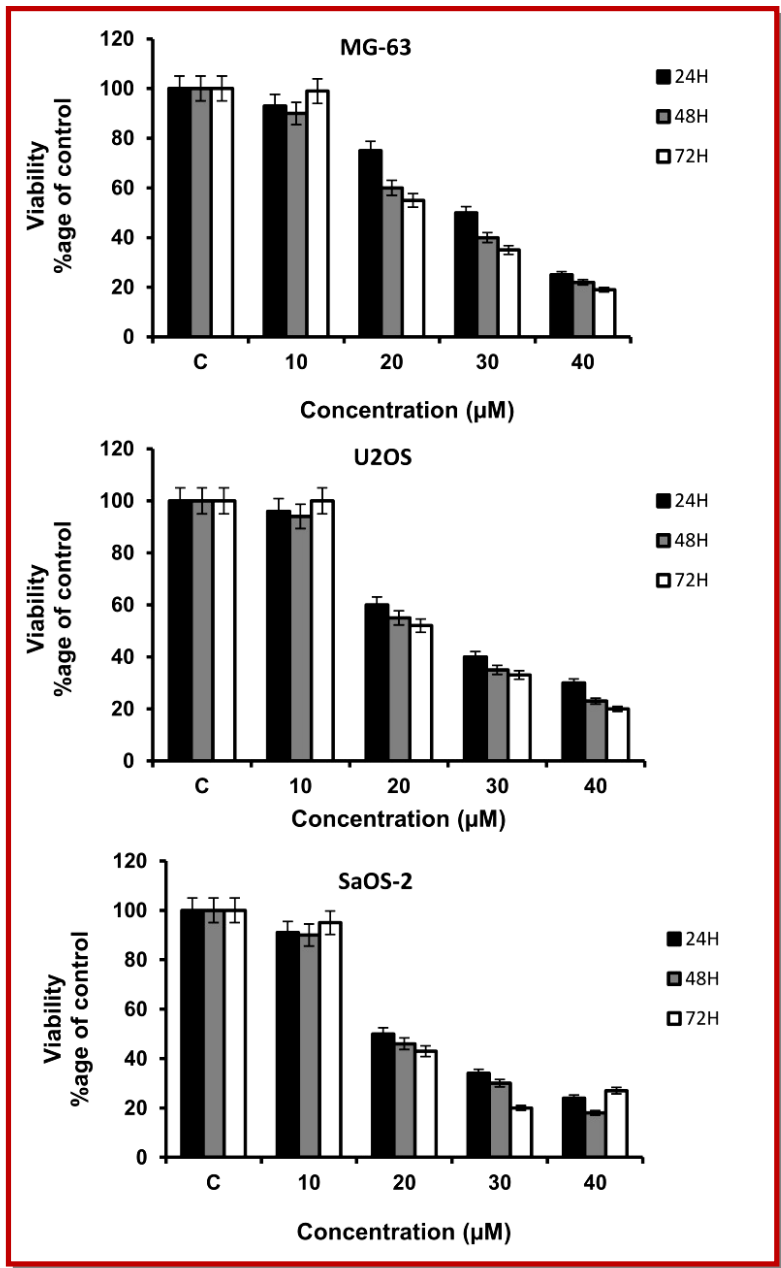

Figure 1: Effect of withaferin A on osteosarcoma cell viability was tested by MTT assay

U2OS, SaOS-2 and MG-63 cells were treated with various concentrations of withaferin A for 24, 48 or 72 hours (control: cells treated with dimethylsulfoxide). MTT assays were used to establish an optimum concentration range of withaferin A for subsequent experiments. Treatment of osteosarcoma cells with withaferin A resulted in cell viability inhibition in a dose- and time-dependent manner. Treatment of osteosarcoma cells with withaferin A beyond $30 \mu \mathrm{M}$ at 24,48 and 72 hours resulted in statistically significant toxicities. Cell viability in withaferin A-treated cells was expressed as the percentage of dimethylsulfoxidetreated samples. Results represent the mean \pm SD of three individual experiments having six determinations per experiment for each experimental condition 


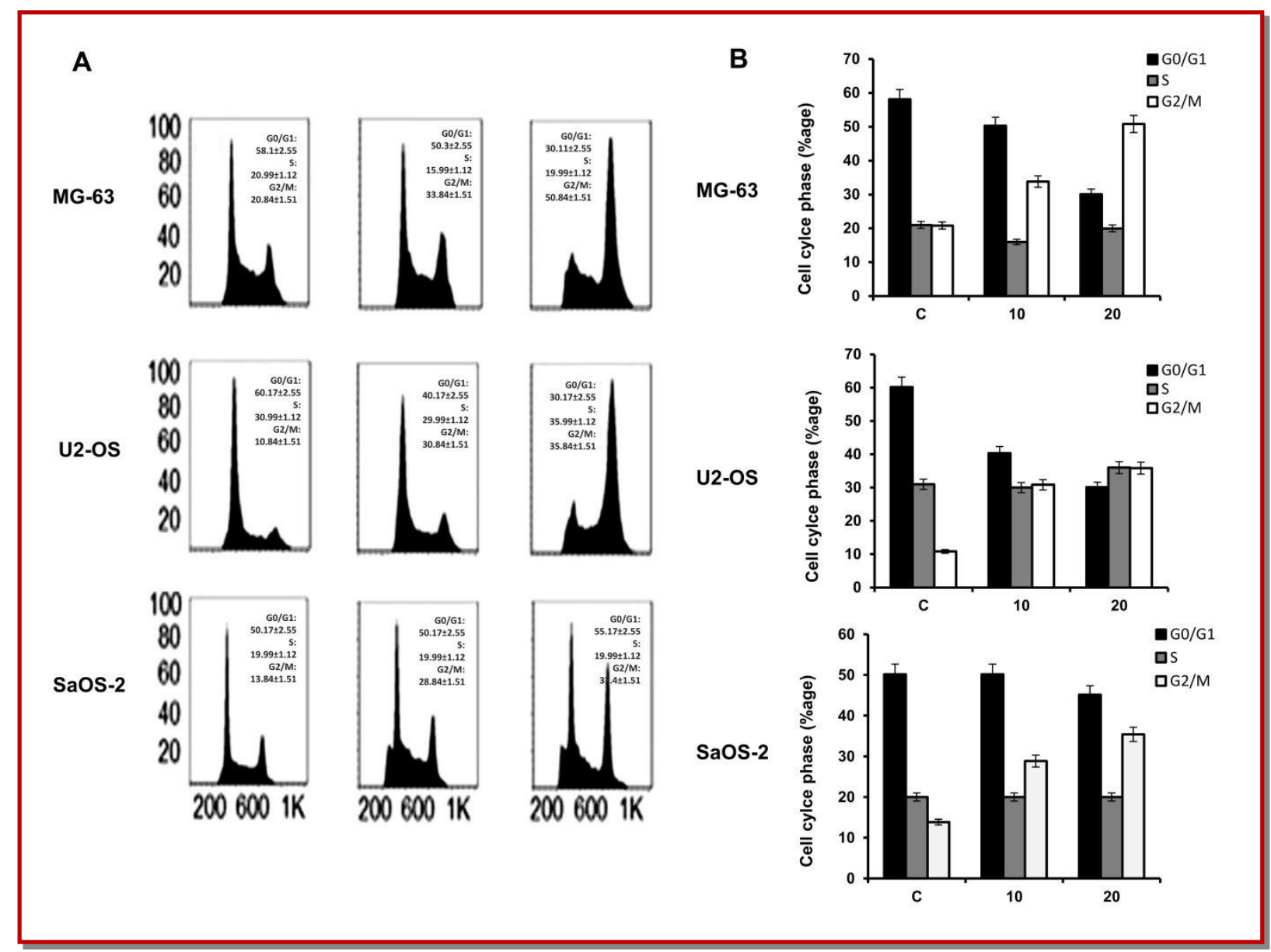

Figure 2: Withaferin A-induced G2 M phase arrest in osteosarcoma cells was measured with cell cycle distribution by flow cytometry analysis

C, control; 10, $20 \mu \mathrm{M}$ withaferin A. U2OS, SaOS-2 and MG-63 cells were treated with 10 and $20 \mu \mathrm{M}$ withaferin A for 48 hours (control: cells treated withdimethylsulfoxide). Treatment with withaferin A resulted in an increase in the number of cells in the G2/M phase and a decrease in the number of cells in the G0/G1 phase, indicating a withaferin A-induced G2M phase arrest. Results are plotted as the percentage of cells in each cell cycle phase. Data are presented as mean \pm SD of three independent experiments

cells and thus indicated a cytotoxic effect of withaferin A on osteosarcoma cells. To further investigate whether withaferin A was inducing cell cycle arrest of osteosarcoma cells, a cell cycle analysis was conducted. Cell cycle distribution of U2OS, SaOS-2 and MG-63 cells, following a 48 hours treatment with $15 \mathrm{~mL}$ of withaferin A, was determined by propidium iodide (PI) staining and flow cytometry. As seen in Figure 2, treatment with withaferin A resulted in an increase in the number of cells in the G2M phase and a decrease in the number of cells in the G0/G1 phase, indicating a withaferin A-induced G2M phase arrest. These data suggested that the decreased proliferation in withaferin A-treated osteosarcoma cells is at least partially a result of the cell cycle arrest by withaferin A.

To understand further the molecular mechanism involved in withaferin A-induced proliferation inhibition, alterations in the cell survival Notch pathway were investigated. Notch-1 and its target genes Hes-1, Hey-1, Hey-2 expression in U2OS and MG-63 cells treated with increasing concentrations of withaferin A for 48 hours were assessed using real-time RT-PCR analysis. Compared with control, there was a reduction of Notch-1, Hes-1, Hey-1 and Hey-2 mRNA levels after withaferin A treatment, suggesting that withaferin A resulted in the transcriptional inactivation of Notch-1 signaling pathway in osteosarcoma cells (Figure 3A). It is important to note that the active functional form of Notch is Notch-1 intracellular domain. Therefore, we focused our studies on Notch-1 intracellular domain at the protein level and Notch-1 in all figure legends means the active functional form of Notch-1. In agreement with the real-time RT-PCR data, western blot showed that Notch-1 protein levels were down-regulated in both U2OS and MG-63 cells treated with withaferin A in a dose-dependent manner (Figure 3B). Together, we concluded that withaferin A regulated the transcription and translation of the Notch-1 gene. To confirm the inactivation of Notch-1 signaling by witha-ferin $\mathrm{A}$, we also detected the other Notch downstream target genes 
A
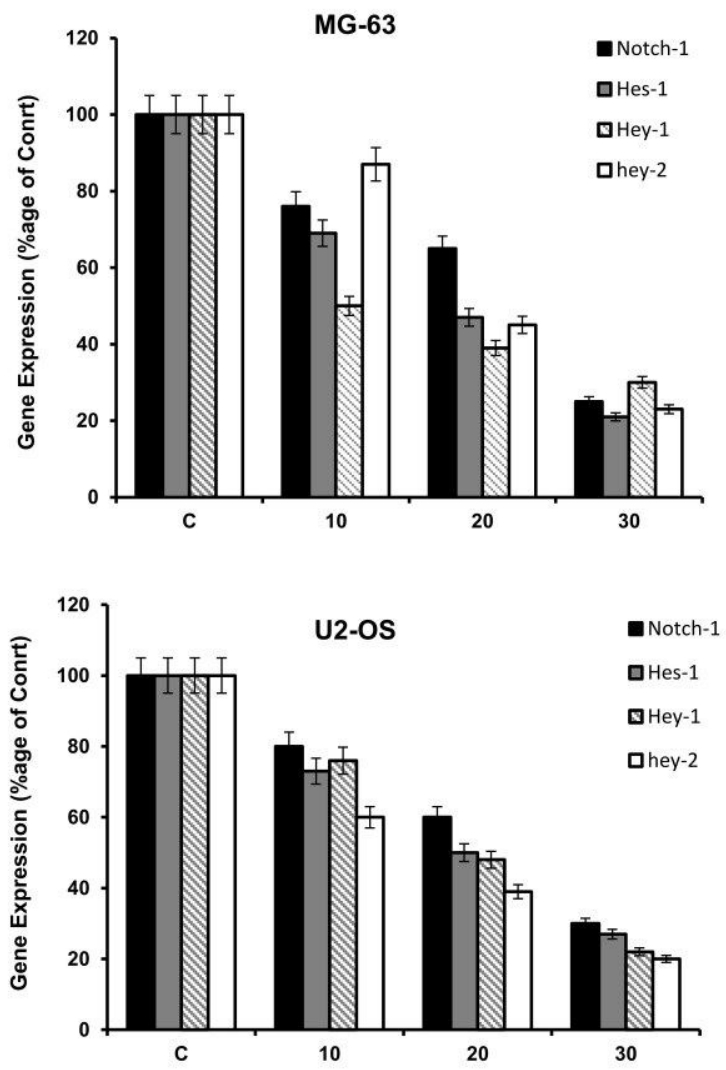

B

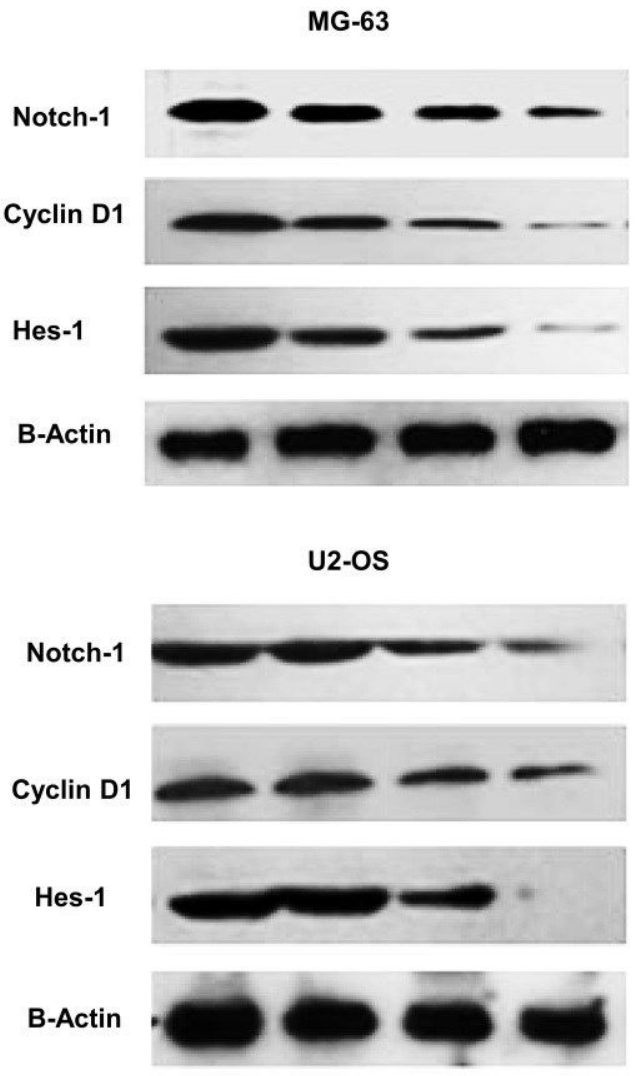

Figure 3: Down-regulation of Notch-1 expression by withaferin A treatment

U2OS and MG-63 cells were treated with 10, 20 and $30 \mu \mathrm{M}$ withaferin A for 48 hours (control: cells treated with dimethylsulfoxide). (A) The mRNA levels in U2OS and MG-63 cells treated with withaferin A were assessed by using real-time RT-PCR analysis. GAPDH was used to normalize the mRNA level. The results are presented as the percentage relative to control. The expression of Notch-1, Hes-1, Hey-1 and Hey-2 genes at the mRNA levels was down-regulated after withaferin A treatment. (B) The protein levels in U2OS and MG-63 cells treated with withaferin A were assessed by western blot analysis. $\beta$-Actin was used as a sample loading control. 'Notch-1' indicates the Notch-1 intracellular domain. The expression of Notch-1, Hes- 1 and cyclin D1 at the protein levels was down-regulated after withaferin A treatment. Data are expressed as mean \pm SD from three independent experiments

such as Hes-1 and cyclin D1. The results demonstrated that the expression of Hes-1 and cyclin D1 was also inhibited by treatment with withaferin A (Figure 3B).

To explore whether the downstream effect of withaferin A induced by down-regulation of Notch-1 was associated mechanistically with MMP induction, we conducted real-time RT-PCR and western blot analysis to detect the alterations in the expression of MMP-2 and MMP-9. The results showed that, compared with control cells, both MMP- 2 and MMP-9 mRNA and protein levels were dramatically down-regulated in withaferin A-treated U2OS and MG-63 cells, respectively. Withaferin A decreased MMP-2 and MMP-9 expression in a dose-dependent manner (Figure 4A, B). These data indicated that withaferin A inhibited the expression and activities of MMP-2 and MMP-9 in osteosarcoma cells.

\section{Discussion}

In the present study, we showed that withaferin A elicited a dramatic effect on proliferation inhibition and G2M cell cycle arrest in U2OS, SaOS-2 and MG-63 osteosarcoma cells, as demonstrated by the MTT assay and flow cytometry analysis, respectively. These results are consistent with the data published by other groups (Koduru et al., 2010). To elucidate the molecular mechanisms of growth and invasion inhibition by withaferin A, we investigated the activity of Notch-1, which plays a key role in the process of cell survival, invasion and metastasis (Zhang et al., 2008; Wang et al., 2008; Hughes, 2009). Aberrant activation of the Notch-1 signaling pathway promotes a myriad of cellular activities such as cell survival, proliferation, migration and invasion, and suppresses apoptosis through a growth-factor mediated survival pathway in many 
A
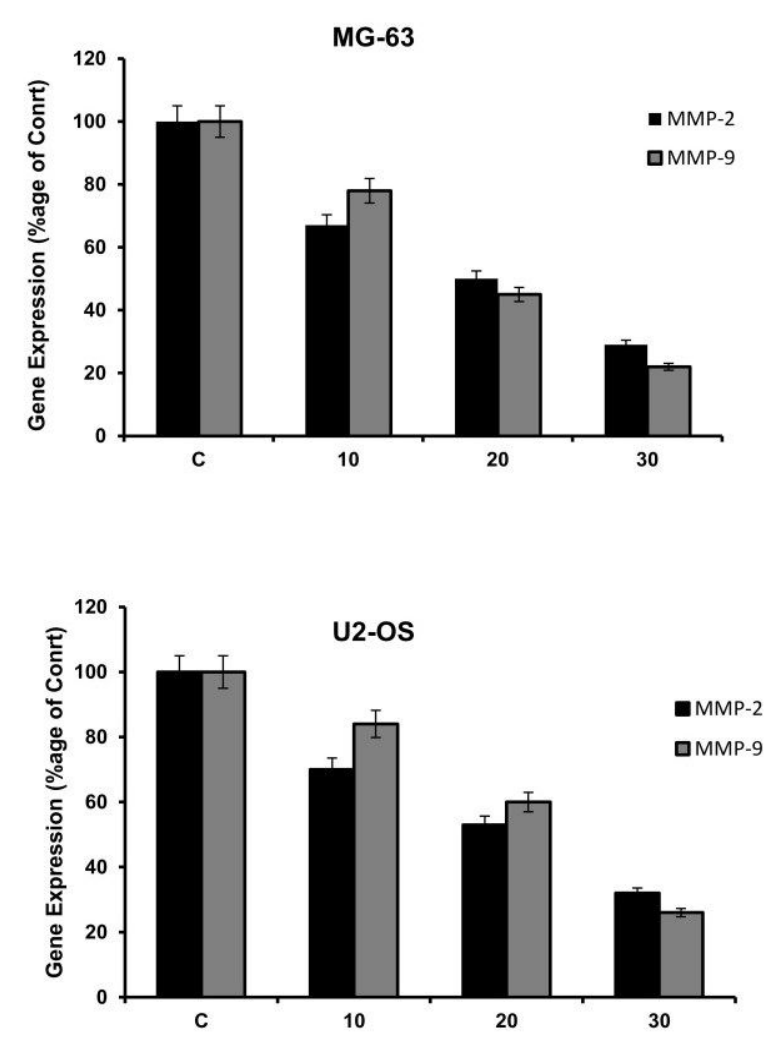

B

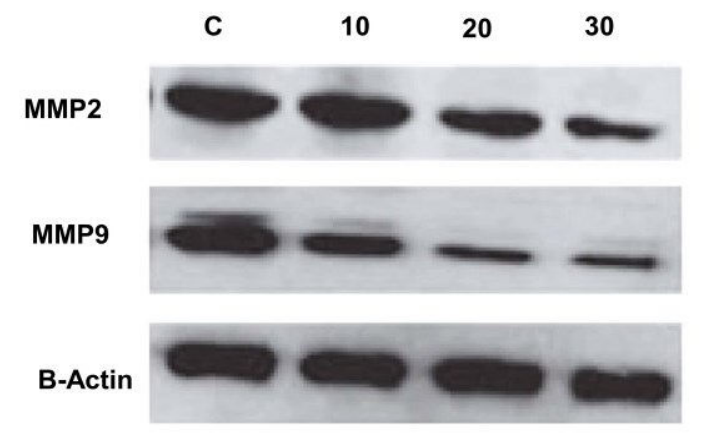

C

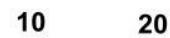

30

MMP2

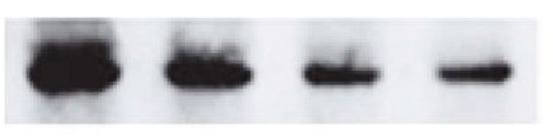

MMP9

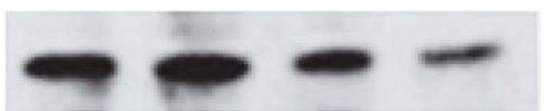

B-Actin

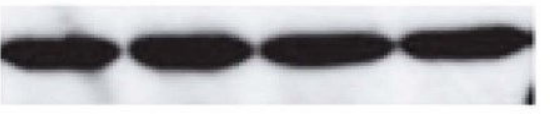

Figure 4: The mRNA (A) and protein levels (B) in withaferin A-treated cells were assessed using real-time RT-PCR and western blot analysis, respectively

GAPDH was used to normalize the mRNA level. The results are presented as the percentage relative to control. $\beta$-Actin was used as a sample loading control. The expression of MMP-2 and MMP-9 genes both at the mRNA and protein levels was dose-dependently reduced with withaferin A treatment

human malignancies, including osteosarcoma (Zhang et al., 2008; Wang et al., 2008; Engin et al., 2009; Tanaka et al., 2009; Hughes, 2009). There is evidence that Notch pathway components and its target genes are upregulated in human pancreatic cancer cells and inactivation of Notch-1 by siRNA induced down-regulation of Bcl-2, Bcl-XL, cyclin A, cyclin D1 and cyclindependent kinase 2, as well as up-regulation of p21 and p27, resulting in cell growth inhibition and apoptosis (Wang et al., 2008). Jang et al. also reports that Notch-1 expression regulates cell death through both apoptosis and cell cycle pathways in erythroleukemia cells and is associated in part with the regulation of Bcl-XL, p21, p27 and NF-jB. Recent results have shown that Notch signaling is involved in osteosarcoma cell survival and contributes to the pathogenesis of human osteosarcoma. Inhibition of the Notch pathway by c-secretase inhibitor treatment prevents osteosarcoma growth in vitro and in vivo by cell cycle regulation (Engin et al., 2009). In addition, Zhang et al. reveals a specific and essential role of Notch signaling in promoting osteosarcoma invasiveness and metastasis in vitro and in vivo.
Therefore, inhibition of Notch signaling may have important therapeutic applications for treating and preventing osteosarcoma metastasis. Recently, withaferin A has been shown to possess potent antineoplastic activity in vitro and in vivo against a variety of malignancies, including prostate, breast and colon cancer (Srinivasan et al., 2007; Stan et al., 2008; Koduru et al., 2010). Withaferin A is known to target multiple biochemical pathways, such as those mediated by NFjB, AKT, JAK/STAT, the Wnt b-catenin pathway and growth (Ichikawa et al., 2006).

In summary, we have presented experimental evidence that supports the role of withaferin A as an antitumor agent. On the basis of our results, we propose a hypothesis that withaferin A-mediated inhibition of cell growth and invasion is associated with inactivation of Notch-1 and its downstream target gene MMPs. The molecular mechanisms by which withaferin A exerts its antitumor activity in osteosarcoma cells, as revealed in our study, has opened up exciting new avenues for devising novel therapeutic strategies. The down-regula- 
tion of Notch-1 and MMPs by withaferin A may be a useful strategy for chemosensitization of metastatic osteosarcoma cells to standard therapeutics.

\section{References}

Arndt CA, Crist WM. Common musculoskeletal tumors of childhood and adolescence. N Engl J Med. 1999; 341: 342-52.

Bielack SS, Kempf-Bielack B, Delling G, Exner GU, Flege S, Helmke K, Kotz R, Salzer-Kuntschik M, Werner M, Winkelmann W, Zoubek A, Jurgens H, Winkler $\mathrm{K}$. Prognostic factors in high-grade osteosarcoma of the extremities or trunk: An analysis of 1,702 patients treated on neoadjuvant cooperative osteosarcoma study group protocols. J Clin Oncol. 2002; 20: 776-90.

Engin F, Bertin T, Ma O, Jiang MM, Wang L, Sutton RE, A Donehower L, Lee B. Notch signaling contributes to the pathogenesis of human osteosarcomas. Hum Mol Genet. 2009; 18: 1464-70.

Hughes DP. How the NOTCH pathway contributes to the ability of osteosarcoma cells to metastasize. Cancer Treat Res. 2009; 152: 479-96.

Ichikawa H, Takada Y, Shishodia S, Jayaprakasam B, Nair MG, Aggarwal BB. Withanolides potentiate apoptosis, inhibit invasion, and abolish osteoclastogenesis through suppression of nuclear factor-kappaB (NF-jB) activation and NF-jB-regulated gene expression. Mol Cancer Ther. 2006; 5: 1434-45.

Jang MS, Miao H, Carlesso N, Shelly L, Zlobin A, Darack N et al.Notch-1 regulates cell death independently of differentiation in murine erythroleukemia cells through multiple apoptosis and cell cycle pathways. J Cell Physiol. 2004; 199: 418-33.

Jayaprakasam B, Nair MG. Cyclooxygenase-2 enzyme inhibitory withanolides from Withania somnifera leaves. Tetrahedron 2003; 59: 841-49.

Kaileh M, Berghe WV, Heyerick A, Horion J, Piette J, Libert C, et al. Withaferin a strongly elicits IkappaB kinase beta hyperphosphorylation concomitant with potent inhibition of its kinase activity. J Biol Chem. 2007; 282: 4253-64.
Katoh M. Notch signaling in gastrointestinal tract (review). Int J Oncol 2007; 30: 247-51

Leong KG, Karsan A. Recent insights into the role of Notch signaling in tumorigenesis. Blood 2006; 107: 2223-33.

Koduru S, Kumar R, Srinivasan S, Evers MB, Damodaran C. Notch-1 inhibition by withaferin-A: A therapeutic target against colon carcinogenesis. Mol Cancer Ther. 2010; 9: 20210.

Li JL, Harris AL. Notch signaling from tumor cells: A new mechanism of angiogenesis. Cancer Cell. 2005; 8: 1-3.

Petrilli AS, de Camargo B, Filho VO, Bruniera P, Brunetto AL, Jesus-Garcia R, et al. Results of the Brazilian osteosarcoma treatment group studies III and IV: Prognostic factors and impact on survival. J Clin Oncol. 2006; 24: 1161-68

Ramdass B, Maliekal TT, Lakshmi S, Rehman M, Rema P, Nair $\mathrm{P}$, et al. Coexpression of Notch1 and NF-kappaB signaling pathway components in human cervical cancer progression. Gynecol Oncol. 2007; 104: 352-61.

Srinivasan S, Ranga RS, Burikhanov R, Han SS, Chendil D. Par4-dependent apoptosis by the dietary compound withaferin A in prostate cancer cells. Cancer Res. 2007; 67: 246-53.

Stan SD, Hahm ER, Warin R, Singh SV. Withaferin A causes FOXO3a- and Bim-dependent apoptosis and inhibits growth of human breast cancer cells in vivo. Cancer Res. 2008; 68: 7661-69.

Subbaraju GV, Vanisree M, Rao CV, Sivaramakrishna C, Sridhar P, Jayaprakasam B, et al. Ashwagandhanolide, a bioactive dimeric thiowithanolide isolated from the roots of Withania somnifera. J Nat Prod. 2006; 69: 1790-92.

Tanaka M, Setoguchi T, Hirotsu M, Gao H, Sasaki H, Matsunoshita Y, et al. Inhibition of Notch pathway prevents osteosarcoma growth by cell cycle regulation. Br J Cancer 2009; 100: 1957-65.

Wang Z, Zhang Y, Li Y, Banerjee S, Liao J, Sarkar FH. Downregulation of Notch-1 contributes to cell growth inhibition and apoptosis in pancreatic cancer cells. Mol Cancer Ther. 2006; 5: 483-93.

Zhang P, Yang Y, Zweidler-McKay PA, Hughes DP. Critical role of Notch signaling in osteosarcoma invasion and metastasis. Clin Cancer Res. 2008; 14: 2962-69. 